ARTICLE

https://doi.org/10.1038/s41467-018-07879-3

\title{
A natural in situ fabrication method of functional bacterial cellulose using a microorganism
}

\author{
Minghong Gao', Juan $\mathrm{Li}^{2}$, Zixian Bao ${ }^{1}$, Moudong $\mathrm{Hu}^{2}$, Rui Nian${ }^{1}$, Dexin Feng${ }^{1}$, Dong $\mathrm{An}{ }^{1}$, Xing $\mathrm{Li}^{1}$, \\ Mo Xian $^{1} \&$ Haibo Zhang ${ }^{1}$
}

The functionalization methods of materials based on bacterial cellulose (BC) mainly focus on the chemical modification or physical coating of fermentation products, which may cause several problems, such as environment pollution, low reaction efficiency and easy loss of functional moieties during application. Here, we develop a modification method utilizing the in situ microbial fermentation method combined with 6-carboxyfluorescein-modified glucose (6CF-Glc) as a substrate using Komagataeibacter sucrofermentans to produce functional BC with a nonnatural characteristic fluorescence. Our results indicate that the microbial synthesis method is more efficient, controllable and environmentally friendly than traditional modification methods. Therefore, this work confirms that BC can be functionalized by using a microbial synthesis system with functionalized glucose, which provides insights not only for the functionalization of $\mathrm{BC}$ but also for the in situ synthesis of other functional materials through microbial synthetic systems.

\footnotetext{
${ }^{1}$ CAS Key Laboratory of Biobased Materials, Qingdao Institute of Bioenergy and Bioprocess Technology, Chinese Academy of Sciences, No. 189 Songling Road, Qingdao 266101, China. ${ }^{2}$ College of Chemistry and Chemical Engineering, Ocean University of China, No. 238 Songling Road, Qingdao 266003 , China. These authors contributed equally: Minghong Gao, Juan Li, Zixian Bao Correspondence and requests for materials should be addressed to M.X. (email: xianmo@qibebt.ac.cn) or to H.Z. (email: zhanghb@qibebt.ac.cn)
} 
$\mathrm{F}$ unctional materials constitute an extremely active and breakthrough research area in which all aspects of materials science are involved, including chemistry, physics, engineering, biology, and nanotechnology. Different forms of functional materials have been developed for various purposes, such as environmental monitoring, food industry, ion battery fabrication, and clinical therapy ${ }^{1-6}$. Of them, functional materials based on bacterial cellulose (BC) have attracted increasing attention because of its excellent physical and chemical properties, including high safety, low cost, high flexibility and adaptability, hydrophilicity, transparency, excellent biocompatibility and biodegradability 7,8 . BC is a porous network-like nanoscale biopolymer produced by the fermentation of microorganisms, which consists of chains of $\beta$-D-glucose linked by $\beta$-1,4-glycosidic bonds. During the fermentation, glucose is metabolized by microorganisms and forms into linear $\beta$-1,4-glucan chains. These cellulose linear chains are secreted extracellularly and crystallized to form cellulose monofilaments. A certain number of monofilaments aggregate to form filamentous fibers (like ribbons), which further form a three-dimensional and gelatinous structure on the surface of a liquid medium ${ }^{9-11}$. The bacterial strains that produce BC include Acetobacter, Rhizobium, Xanthococcus, Pseudomonas, Azotobacter, Aerobacter, and Alcaligenes. Among them, Komagataeibacter xylinum (formerly Gluconacetobacter xylinum) is the most commonly used microorganism to synthesize $\mathrm{BC}$ due to its high yield. In our work, Komagataeibacter sucrofermentans (Komagataeibacter xylinum subsp. sucrofermentans, K. sucrofermentans) was used for BC production due to its ability to synthesize cellulose stably and produce thick pellicles under static growth conditions ${ }^{12}$.

BC has several advantages over plant-derived cellulose, including high purity (free of lignin, hemicellulose and pectin) ${ }^{13}$, high crystallinity, high elasticity and conformability, low density, high specific surface area, high degree of polymerization, excellent permeability, high porosity and water content, and high mechanical strength in wet state $e^{7,10,14-17}$, ensuring the multifunction of BC. More importantly, BC is superior in terms of modification. First, it can be shaped in situ during fermentation to form different structures, such as tubes, membranes, spheres and layers of thin fibers ${ }^{13}$, meeting the needs of functional materials for various applications with a one-step synthesis. Second, BC has a multihydroxyl molecular structure that can be functionalized, such as by functional compound modification or polymer coating ${ }^{18}$. As a result, functionalized BC-based materials have emerged and are utilized in various fields, such as chemical sensing, bioimaging, UV screening, oil adsorption, fuel cells, biomedical materials, ion detection, and anticounterfeiting labels $2,6,19-22$.

$\mathrm{BC}$ is often functionalized with chemical modification or physical coating based on fermentation products. Commonly used modification methods include nanoparticle coating, metal oxide modification, fluorescent substance modification and ionic liquid replacement ${ }^{2,23-25}$. The physical coating can provide a mild modification condition, but functional moieties potentially suffer from shedding due to weak physical interactions. The high polarity and strong intermolecular hydrogen bonding within $\mathrm{BC}$ lead to its poor solubility in water and standard organic solvents ${ }^{26}$. Therefore, although BC has a multihydroxyl molecular structure, the chemical modification of cellulose-based materials with functional groups remains plagued by a limited choice of solvents, such as N,Ndimethylacetamide/LiCl, dimethyl sulfoxide/tetrabutylammonium fluoride, $\mathrm{N}$-methylmorpholine-N-oxides and ionic liquids ${ }^{27,28}$. However, the utilization of these toxic chemical agents has serious negative effects on the environment, simultaneously influences the green safety of products, and adds complexity to waste discharge treatment ${ }^{29}$, limiting its large-scale production. Moreover, chemical modification can be performed with cellulose nanocrystals or nanofibers in a heterogeneous system ${ }^{2}$, which may lead to a low reaction efficiency. More importantly, due to the multihydroxyl molecular structure of $\mathrm{BC}$, selective modification on a specific $\mathrm{C}$ site of $\mathrm{BC}$ is difficult using chemical methods, whereas the biological synthesis method with high specificity may solve this problem. However, no functionalized modifications on BC using a microbial in situ fermentation system were reported, which may provide a potential method for biological modification of BC, with the dual goal of providing an innovative microbial modification method that circumvents the limitations of chemical and physical modification, and understanding the microbial metabolic pathways that produce functionalized products.

Recently, an innovative method for the synthesis of functional plant cellulose was reported. Gossypium hirsutum fertilized ovules were selected as the in vitro fabrication condition to perform in situ functionalization of cellulose fibers ${ }^{30}$. Using glucose as the carrier, functional molecules were introduced into plant cellulose fibers through the cotton ovule. Through the regulation of glucose and functional groups, cotton fibers with fluorescent and magnetic properties were prepared, respectively. The results indicated that the fluorescent cotton fiber is more durable and environmentally friendly than those prepared with the conventional method of compound coating modification ${ }^{30}$. A microbial fermentation system has several advantages over other synthesis methods, such as high efficiency, short production cycle, and ease of regulating the functionality of products. Therefore, this system is an optical research platform for synthesizing functional biobased materials ${ }^{31}$. However, whether this in situ modification method can be extended to other biosynthesis systems, particularly high-efficiency microbial systems, remains to be elucidated.

Here, we perform an innovative modification method for functional BC biofabrication using a microbial system. Glucose is functionally modified with 6-carboxyfluorescein (6CF) and used as a substrate to produce the functional BC by in situ fermentation with $K$. sucrofermentans. The fluorescence intensity of functional $\mathrm{BC}$ can be controlled by adjusting the concentration of 6CF-modified glucose (6CF-Glc) in the culture medium. Our results verify that $\mathrm{BC}$ could be functionally modified by a microbial synthesis system, which has superiority over chemical modification. Therefore, our approach has implications for both the modification of $\mathrm{BC}$ and production of other valuable functional materials via biosynthetic systems.

\section{Results}

Chemical preparation of functional BC. We used a green chemical method, in which cellulose-based materials were modified with fluorophores by simple immersion followed by drying above $80^{\circ} \mathrm{C}$ for the preparation of functional $\mathrm{BC}^{29}$, and retouching of the BC with 6CF (a fluorescent dye) (Fig. 1a). Finally, we obtained the chemically modified functional $\mathrm{BC}(\mathrm{Ch}-6 \mathrm{CF} / \mathrm{BC})$. The green fluorescence of Ch-6CF/BC was observed under ultraviolet (UV) light $(365 \mathrm{~nm})$ (Supplementary Figure 1). Fourier transform infrared spectroscopy with attenuated total reflectance (FT-IR ATR) analysis of the $\mathrm{Ch}-6 \mathrm{CF} / \mathrm{BC}$ fibers showed no absorption peak at $1740 \mathrm{~cm}^{-1}(\mathrm{C}=\mathrm{O}$ stretching in ester $)$, which was the same as the FT-IR ATR spectrum of BC (Supplementary Figure 2).

Biological preparation of functional BC. Based on the biosynthesis of functional plant cellulose ${ }^{30}, 6 \mathrm{CF}-\mathrm{Glc}$ was used as a substrate to produce biologically modified BC (6CF-BC) by in situ fermentation of $K$. sucrofermentans. The microorganism was sensitive to the additive molecules in the medium; therefore, we first explored the selectivity of $6 \mathrm{CF}$ on $K$. sucrofermentans. $6 \mathrm{CF}$ was added directly to the fermentation medium, and K. sucrofermentans was cultured under standard conditions. 
a
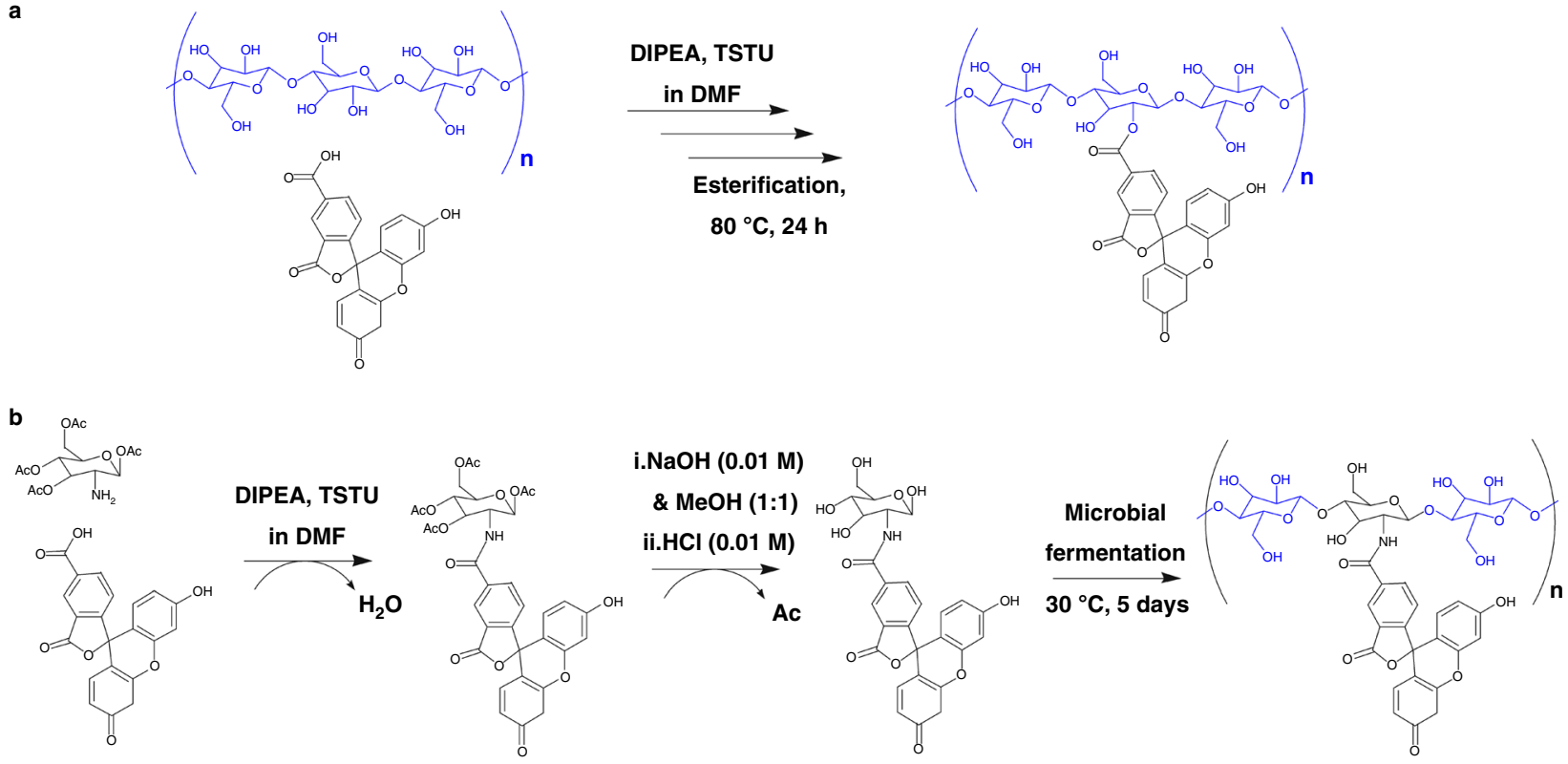

Fig. 1 Chemical and biological preparation of functional BC. a Chemically modified BC (Ch-6CF/BC) was prepared by soaking neat BC in activated 6CF solutions for $30 \mathrm{~min}$ at room temperature under inert conditions and drying at $80^{\circ} \mathrm{C}$. b The 6 -carboxyfluorescein-glucose (6CF-Glc) was first synthesized starting from 6-CF and 1,3,4,6-tetra-O-acetyl-2-amino-2-deoxy- $\beta$-D-glucopyranose, and it was then used as a carbon source to produce functionalized 6 CF-BC through microbial fermentation

A complete $\mathrm{BC}$ pellicle was produced on the surface of the culture medium (Supplementary Figure 3). The obtained BC was observed with confocal laser scanning microscopy (CLSM, emission wavelength, $488 \mathrm{~nm}$ ), and $K$. sucrofermentans on the surface of BC exhibited fluorescence (Supplementary Figure 4), demonstrating that $6 \mathrm{CF}$ entered the cell body of $K$. sucrofermentans and had no clear impact on its growth and metabolism. Thus, the synthesis of fluorescent $\mathrm{BC}$ through the fermentation of $K$. sucrofermentans with 6CF-Glc as the carbon source is feasible. $6 \mathrm{CF}$-Glc was synthesized by reacting the free amino group located at $\mathrm{C} 2$ of the acetyl-protected glucosamine moiety with the carboxylate from $6 \mathrm{CF}$ (Fig. 1b). The UV-Vis spectrum, mass spectrometry, and ${ }^{1} \mathrm{H}$ NMR were further used to verify the successful synthesis of 6CF-Glc (Supplementary Figure 5).

6CF-BC was obtained by adding 6CF-Glc to the culture medium of $K$. sucrofermentans. Based on the feeding ratios of 6CF-Glc, the obtained 6CF-BC was divided into two groups, HC-6CF-BC (high concentration of 6CF-Glc, $0.95 \mathrm{mg} \mathrm{mL}^{-1}$ ) and LC-6CF-BC (low concentration of $6 \mathrm{CF}-\mathrm{Glc}, 0.38 \mathrm{mg} \mathrm{mL}$ ${ }^{-1}$ ). For contrast, the neat $\mathrm{BC}$ was immersed in a sterile fermentation medium containing 6CF-Glc and allowed physical adsorption (Im-6CF/BC). The synthesis images of 6CF-BC with different concentrations of 6CF-Glc from days 2 to 5 are shown in Fig. 2a,b. The thickness of HC-6CF-BC (Fig. 2a) was thinner than LC-6CF-BC (Fig. 2b), indicating that the structure of $\mathrm{BC}$ was affected by the increasing concentration of 6CF-Glc. We proposed that 6CF-Glc impeded the formation of intermolecular hydrogen bonds between cellulose and water molecules.

To remove the residual medium and microorganisms, the samples, including $\mathrm{BC}, 6 \mathrm{CF}-\mathrm{BC}$ and $\mathrm{Im}-6 \mathrm{CF} / \mathrm{BC}$, were treated with $\mathrm{NaOH}(2 \%, w / v)$ and then observed under UV light and with CLSM. Green fluorescence was observed on 6CF-BC samples (Fig. 2c, f, g), and no fluorescence was found for Im6CF/BC and BC (Fig. 2d, e, h, i). The results showed that 6CF-Glc was integrated into $\mathrm{BC}$ by the metabolism of $\mathrm{K}$. sucrofermentans and further eliminated interference from the fluorescence of 6CF-BC caused by surface contamination. In addition, the fluorescence distribution of HC-6CF-BC was more uniform than that of $\mathrm{Ch}-6 \mathrm{CF} / \mathrm{BC}$ (Supplementary Figure 1). The fluorescent signal of HC-6CF-BC was stronger than that of LC-6CF-BC when observed with CLSM and fluorescence microscopy (Fig. 3 and Supplementary Figure 6), demonstrating that the fluorescence intensity of functional $\mathrm{BC}$ was controlled by adjusting the concentration of $6 \mathrm{CF}-\mathrm{Glc}$ in the culture medium.

Structural characterization of functional BC. FT-IR ATR spectroscopy was performed to identify the modification of BC. Compared with $B C$, new peaks appeared at $1530 \mathrm{~cm}^{-1}(\mathrm{C}=\mathrm{O}$ stretching), $1650 \mathrm{~cm}^{-1}(\mathrm{C}=\mathrm{O}$ stretching in $\mathrm{CO}-\mathrm{NH})$, and $1453 \mathrm{~cm}$ ${ }^{-1}$ (benzene ring vibration) in the spectra of 6CF-BC (Fig. 4a, black and red). Furthermore, a wide absorption band appeared at $3354 \mathrm{~cm}^{-1}$ (OH stretching). The peak at $2904 \mathrm{~cm}^{-1}\left(\mathrm{CH}_{2}\right.$ antisymmetric stretching), an inconspicuous peak at $2853 \mathrm{~cm}^{-1}$ $\left(\mathrm{CH}_{2}\right.$ symmetric stretching), a peak at $1163 \mathrm{~cm}^{-1}$ (anti-symmetric stretching of C-O-C) and a peak at $896 \mathrm{~cm}^{-1}(\beta-1,4$-glycosidic bonds) became sharper $30,32,33$. The shift of peaks to lower wavenumber regions was mainly caused by the abundant inter and intramolecular hydrogen bonds in $\mathrm{BC}^{34}$. Acylamino was the characteristic group representing the combination of $6 \mathrm{CF}$ and glucose. Therefore, the new peaks appeared at $1530 \mathrm{~cm}^{-1}$ and $1650 \mathrm{~cm}^{-1}$, verifying the successful introduction of $6 \mathrm{CF}$ into $\mathrm{BC}$.

The pattern of X-ray diffraction (XRD) (Fig. 4b) showed that all samples had two absorption peaks, appearing at $14.5^{\circ}$ and $22.6^{\circ}$, corresponding to the (110) and (200) planes of the cellulose form I- $\beta$ crystal ${ }^{35,36}$. These results revealed that the introduction of $6 \mathrm{CF}$ had no significant effect on the crystal structure of $\mathrm{BC}$, indicating that $6 \mathrm{CF}$ modification may mainly occur in the amorphous domains of BC. However, the crystallinity of HC6CF-BC (71.8\%) and LC-6CF-BC (90.0\%) was lower than that of BC $(98.3 \%)$, demonstrating that 6CF-Glc interfered with the orderly arrangement of $\mathrm{BC}$ chains and reduced the crystallinity. 


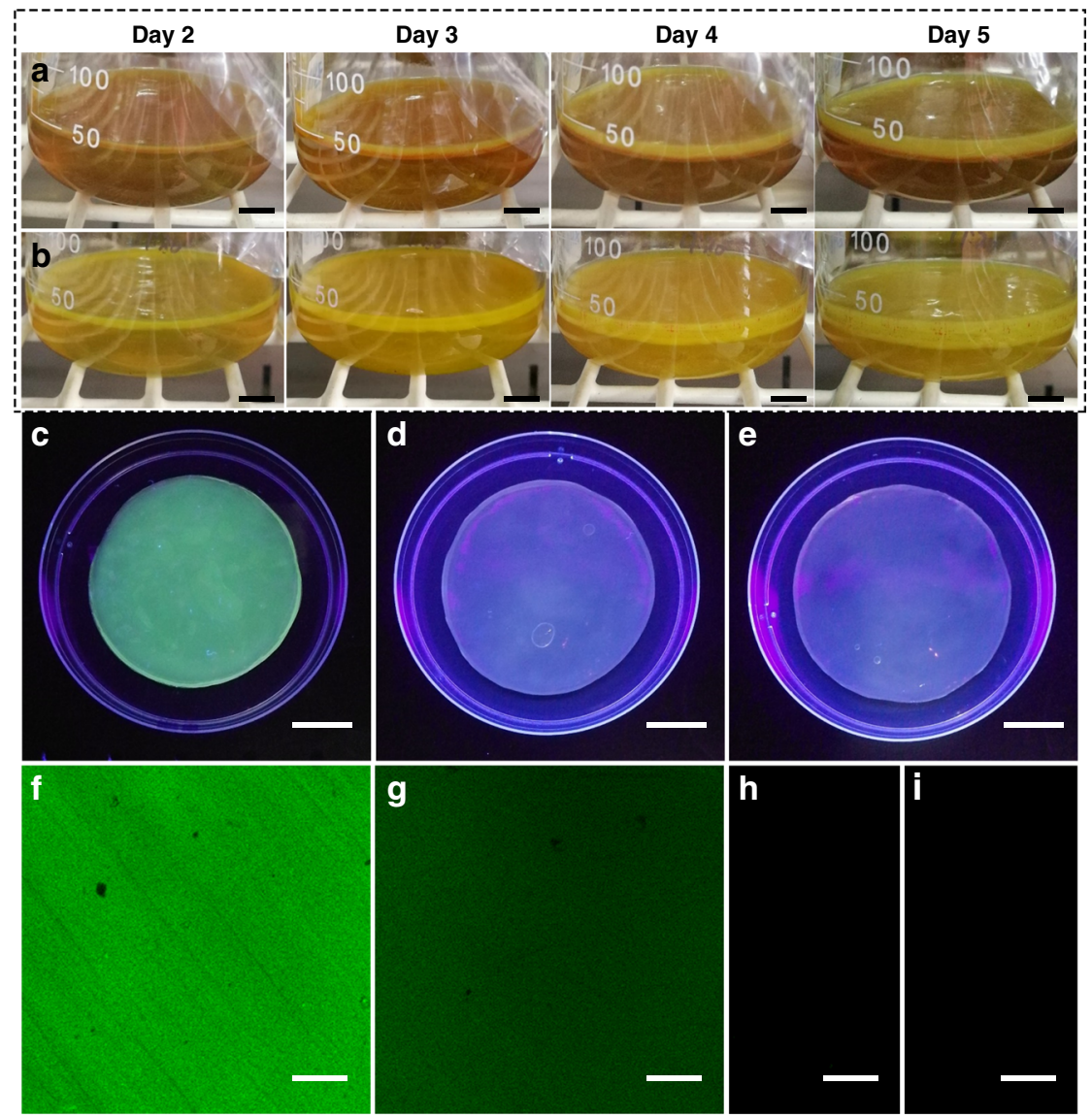

Fig. 2 Biological incorporation of fluorescent-tagged glucose (6CF-Glc) into BC fibers. a, b Images of 6CF-BC fermentation under standard conditions $\left(30^{\circ} \mathrm{C}\right.$ ) on days $2,3,4$, and 5 . The thickness of a HC-6CF-BC (high concentration of 6 CF-Glc, $0.95 \mathrm{mg} \mathrm{mL}^{-1}$ ) was thinner than $\mathbf{b} \mathrm{LC}-6 \mathrm{CF}-\mathrm{BC}$ (low concentration of $6 \mathrm{CF}-\mathrm{Glc}, 0.38 \mathrm{mg} \mathrm{mL}^{-1}$ ). The scale bar corresponds to $1 \mathrm{~cm}$. c-e Images of $6 \mathrm{CF}-\mathrm{BC}, \mathrm{BC}$, and Im-6CF/BC observed under UV light $(365 \mathrm{~nm})$. c 6 CF-BC showed green fluorescence, whereas $\mathbf{d} \mathrm{Im}-6 \mathrm{CF} / \mathrm{BC}$ and $\mathbf{e} \mathrm{BC}$ showed no fluorescence. The scale bar corresponds to $2 \mathrm{~cm}$. $\mathbf{f}-\mathbf{i} \mathrm{Images}$ of 6CF-BC, BC, and Im-6CF/BC observed with CLSM (excitation wavelength $488 \mathrm{~nm}$ ). $\mathbf{f}$ HC-6CF-BC and $\mathbf{g}$ LC-6CF-BC showed green fluorescence, whereas $\mathbf{h} \mathrm{Im}-6 \mathrm{CF} / \mathrm{BC}$ and $\mathbf{i} \mathrm{BC}$ showed no fluorescence. The scale bar corresponds to $100 \mu \mathrm{m}$

Therefore, the crystallinity was decreased with the increasing concentration of 6CF-Glc.

Thermogravimetric (TG) analysis (Fig. 4c) showed that thermal decomposition began at $230^{\circ} \mathrm{C}$ for both HC-6CF-BC (black) and LC-6CF-BC (red), and $300^{\circ} \mathrm{C}$ for BC (blue). The differential thermal gravity (DTG) (Fig. 4d) showed that the lowest endothermic peaks of HC-6CF-BC (black) and LC-6CF$\mathrm{BC}$ (red) were $330^{\circ} \mathrm{C}$ and $335^{\circ} \mathrm{C}$, respectively, and it was $365^{\circ} \mathrm{C}$ for $\mathrm{BC}$ (blue). The maximum weight loss rate occurred at this time, corresponding to pyrolysis of the $\beta$-1,4-glycosidic bond. The endothermic peak of 6 CF-BC was wider (from $220^{\circ} \mathrm{C}$ to $450^{\circ} \mathrm{C}$ ) and less intense, whereas it was sharper (from $280^{\circ} \mathrm{C}$ to $390^{\circ} \mathrm{C}$ ) and stronger in the spectrum of $\mathrm{BC}$. The crystallization temperature of $6 \mathrm{CF}-\mathrm{BC}$ was lower than that of $\mathrm{BC}$ by $30-35^{\circ} \mathrm{C}$ (Fig. 4d), indicating that the thermal stability of $6 \mathrm{CF}-\mathrm{BC}$ was poorer than that of $\mathrm{BC}$ and that the amorphous cellulose content was increased in $6 \mathrm{CF}-\mathrm{BC}^{37}$. These results were consistent with the results of XRD.

Scanning electron micrographs (SEM) showed that there was no significant differences in the diameters of $\mathrm{BC}$ fibers for HC6CF-BC (Fig. 5a), LC-6CF-BC (Fig. 5b), and BC (Fig. 5c). However, the pore size of $\mathrm{BC}$ was enlarged with the increasing concentration of 6CF-Glc. We hypothesized that 6CF-Glc interfered with the arrangement of $\mathrm{BC}$ chains and reduced the interactions of hydrogen bonds among cellulose chains, leading to their loose arrangement and the enhancement of pore size. This suspicion was also confirmed through the cross-sectional SEM of different BC samples (Fig. 5d-f). The gaps of HC-6CF-BC were significantly larger than those of the $\mathrm{BC}$ samples. Moreover, the dried HC-6CF-BC was thicker than the dried LC-6CF-BC and dried BC (Fig. 5g-i). Therefore, 6CF may have a certain influence on the crystal structure and crystallinity of $\mathrm{BC}$. The results were consistent with the XRD and TG analysis.

Finally, we performed tensile tests on BC, LC-6CF-BC,and HC6CF-BC (Supplementary Figure 7 and Supplementary Table 1). The $6 \mathrm{CF}-\mathrm{BC}$ had a lower elastic modulus and tensile strength than BC. Concomitantly, the values of elongation at break were enhanced with the increasing concentration of 6CF-Glc, and HC$6 \mathrm{CF}-\mathrm{BC}$ showed the highest values. Based on the results of XRD, TG, and SEM, the crystallinity of 6CF-BC was reduced by adding $6 \mathrm{CF}-\mathrm{Glc}$, which further influenced the mechanical properties of 6CF-BC.

\section{Discussion}

This study micro-biosynthesizes functional BC successfully. Traditional modifications on BC are mainly conducted with fermentation products using chemical modifications or physical coatings. Traditional physical modification has primarily concentrated on the sputter coating of metal oxide or nanoparticles and evaporation-induced self-assembly based on cellulose nanocrystals ${ }^{24,38}$, which needed complex operations and was not 

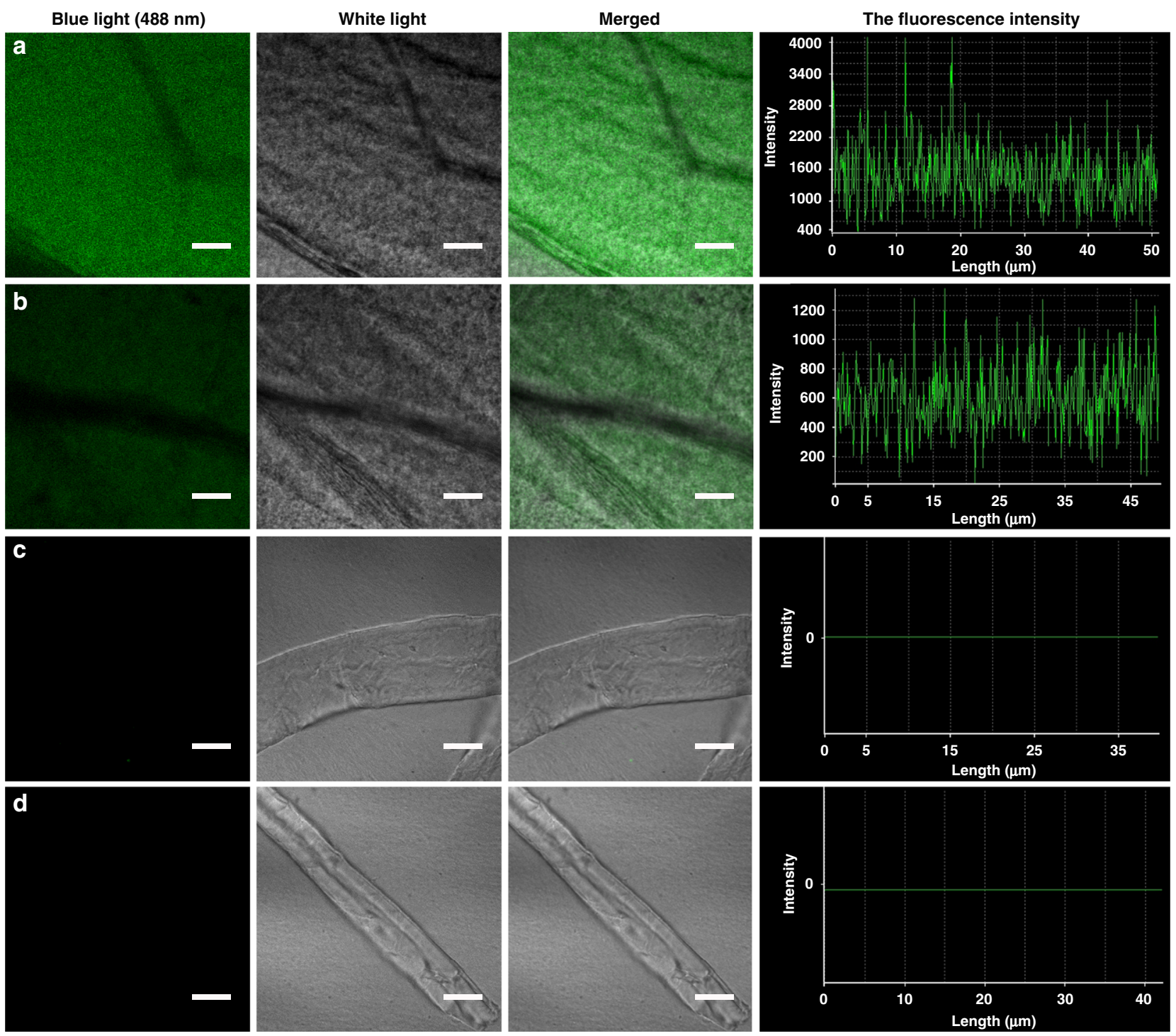

Fig. 3 Detection of fluorescence intensity. The fluorescence intensity of HC-6CF-BC, LC-6CF-BC, Im-6CF/BC and BC was detected using CLSM under the same parameter settings. a HC-6CF-BC; b LC-6CF-BC; c Im-6CF/BC; d BC. The average fluorescence intensities of a HC-6CF-BC and b LC-6CF-BC were 1492 a.u. and 612 a.u., respectively. However, no fluorescence was observed in c Im-6CF/BC and d BC, and both fluorescence intensities were zero. The scale bar corresponds to $10 \mu \mathrm{m}$

suitable for large-scale production. Due to the poor solubility of BC, chemical modification was often conducted with special solvents or in a heterogeneous reaction system based on cellulose nanocrystals, which have several drawbacks, such as low efficiency, waste of raw materials and an increase in the burden of waste discharge treatment ${ }^{29}$. Our work aims to circumvent these limitations. A recent study reported a biosynthesis system that can be utilized to produce functional plant cellulose ${ }^{30}$. The key point is the modification of glucose, which is then used as the carrier to introduce functional moieties into plant cellulose. Glucose is a soluble polysaccharide, which is a carbon source for the synthesis of BC by K. sucrofermentans. Therefore, we anticipate that utilizing the functionalized glucose as the carbon source can help produce BC with tailored properties using a microbial fermentation system.

We modified the glucose with $6 \mathrm{CF}$, and the product (6CF-Glc) was added to the fermentation medium of $\mathrm{K}$. sucrofermentans to synthesize functional $6 \mathrm{CF}-\mathrm{BC}$. We observed that $6 \mathrm{CF}-\mathrm{BC}$ had a green fluorescence based on UV and CLSM detection. We further modified $\mathrm{BC}$ using a chemical method. $\mathrm{Ch}-6 \mathrm{CF} / \mathrm{BC}$ showed green fluorescence, but the fluorescence signal distribution was less uniform than that of $6 \mathrm{CF}-\mathrm{BC}$, which may be related to the heterogeneous reaction system. Unexpectedly, the FT-IR ATR spectrum of $\mathrm{Ch}-6 \mathrm{CF} / \mathrm{BC}$ was consistent with that of $\mathrm{BC}$, and no characteristic absorption peaks appeared at $1740 \mathrm{~cm}^{-1}(\mathrm{C}=\mathrm{O}$ stretching in ester). This result revealed that no ester bonds formed on $\mathrm{Ch}-6 \mathrm{CF} / \mathrm{BC}$. Therefore, the method ${ }^{21}$ we referred to was not suitable for the chemical modification of $\mathrm{BC}$ with $6 \mathrm{CF}$. More importantly, due to the poor solubility of $6 \mathrm{CF}$ in water, the chemical modification to $\mathrm{BC}$ required a large amount of organic solvent (DMF), which not only increased the cost but also caused environmental pollution due to its toxic side effects ${ }^{39}$. Thus, the biological modification method is more suitable than the chemical method for large-scale production. Therefore, the biosynthesis system was more efficient than chemical modification, particularly when modified with water-insoluble materials.

We then tested the structural characteristics of $6 \mathrm{CF}-\mathrm{BC}$, Ch-6CF/BC, and BC. 6CF-BC was synthesized by microbial 

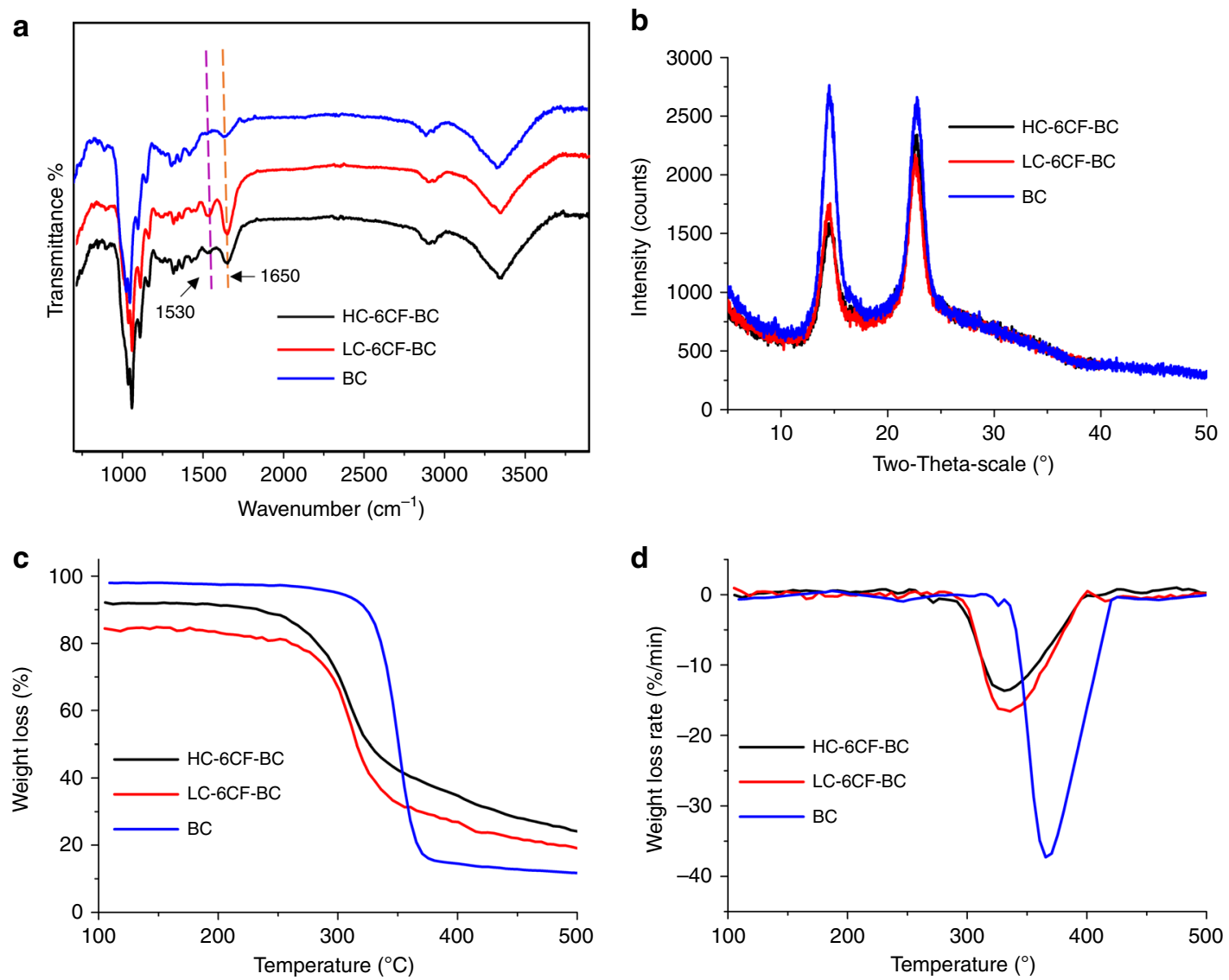

Fig. 4 Structural characterization of different BC samples. a FT-IR ATR spectra of HC-6CF-BC (black), LC-6CF-BC (red) and BC (blue), showing a structural modification. New peaks appeared at $1530 \mathrm{~cm}^{-1}$ and $1650 \mathrm{~cm}^{-1}$ in the spectra of HC-6CF-BC and LC-6CF-BC, demonstrating the successful introduction of $6 C F$ into BC. b XRD spectra of HC-6CF-BC (black), LC-6CF-BC (red) and BC (blue), showing changes in crystallinity. c TG and d DTG analysis of HC-6CF$B C$ (black), LC-6CF-BC (red) and BC (blue). The lowest endothermic peak of HC-6CF-BC (black) and LC-6CF-BC (red) was $330^{\circ} \mathrm{C}$ and $335^{\circ} \mathrm{C}$,

respectively, and the peak was $365^{\circ} \mathrm{C}$ for $\mathrm{BC}$ (blue). The crystallization temperature of $6 \mathrm{CF}-\mathrm{BC}$ was lower than $\mathrm{BC}$ by $30-35^{\circ} \mathrm{C}$, indicating an increase in the amorphous cellulose content in 6CF-BC

metabolism but not through the physical adsorption of 6CF-Glc, which was verified by FT-IR ATR. According to the results of $\mathrm{XRD}$, TG, and SEM, the microstructure of 6CF-BC fibers was maintained and remained type I- $\beta$ cellulose compared to $\mathrm{BC}$. However, due to the addition of $6 \mathrm{CF}$, the regular arrangement among the glucose molecules was disrupted, which in turn reduced the crystallinity and thermal stability of 6CF-BC. Furthermore, with an increase in 6CF-Glc concentration, the tensile strength was also reduced while the strain was increased, which was consistent with the results in a previous study ${ }^{30}$.

The $\mathrm{C} 1$ position is essential for the polymerization of glucose units into fibers ${ }^{30}$. $6 \mathrm{CF}$ was attached to the $\mathrm{C} 2$ position of glucose, leaving the $\mathrm{C} 1$ and $\mathrm{C} 4$ positions free for cellulose formation. Thus, 6CF-Glc could be utilized by K. sucrofermentans. Based on the metabolic pathway of glucose ${ }^{40}$, the metabolism of $6 \mathrm{CF}-\mathrm{Glc}$ was proposed as followed. After 6CF-Glc entered K. sucrofermentans, it might be first phosphorylated by glucokinase to obtain 6CF-Glc-6-phosphate, which might be further converted to $6 \mathrm{CF}-\mathrm{Glc}$-1-phosphate by the isomerization of glucose phosphate isomerase. Then, glucose pyrophosphorylase might convert the 6CF-Glc-1-phosphate to uridine diphosphate-6CFGlc (UDP-6CF-Glc). Finally, UDP-6CF-Glc might be ligated via $\beta$-1,4-glycosidic linkage to synthesize 6CF-BC (Fig. 6). Though the hypothesis of 6CF-Glc metabolism was proposed base on the metabolic pathway of glucose, it remained to be further authenticated.
Overall, our results verified the notion that $\mathrm{BC}$ could be functionalized by using a microbial synthesis system. This method can be extended to the production of other functional materials. The key technologies for this method are (i) the design of metabolic substrates (functionalized carbon and nitrogen sources) and (ii) an understanding of the mechanism of substrate-enzyme recognition and substrate resistance. Based on this premise, the yield can also be improved, and the varieties of metabolized substrates can be expanded. For example, Thongsomboon et al. reported that functionalized cellulose can be naturally produced by modifying the genetically engineered strain $^{41}$. The optimal functionalized method should be (i) green and environmentally friendly, (ii) highly efficient, (iii) low cost, (iv) suitable for large-scale production, and (v) easily modified on specific sites. Therefore, there is a worldwide demand for environmental-friendly and sustainable production of new added-value chemicals and other materials based on biosynthesis methods ${ }^{42,43}$. Functional molecules can be efficiently assembled by utilizing the enzyme catalysis of microbial cell factories, breaking the bottlenecks of physical and chemical synthetic methods and producing functional materials and intermediate chemicals, particularly for those obtained with difficulty by using traditional methods. Therefore, although our data has several limitations, this method meets all five criteria, lending experimental support to our idea of an innovative biosynthesis method to functionalize BC. Our work not only confirmed that 

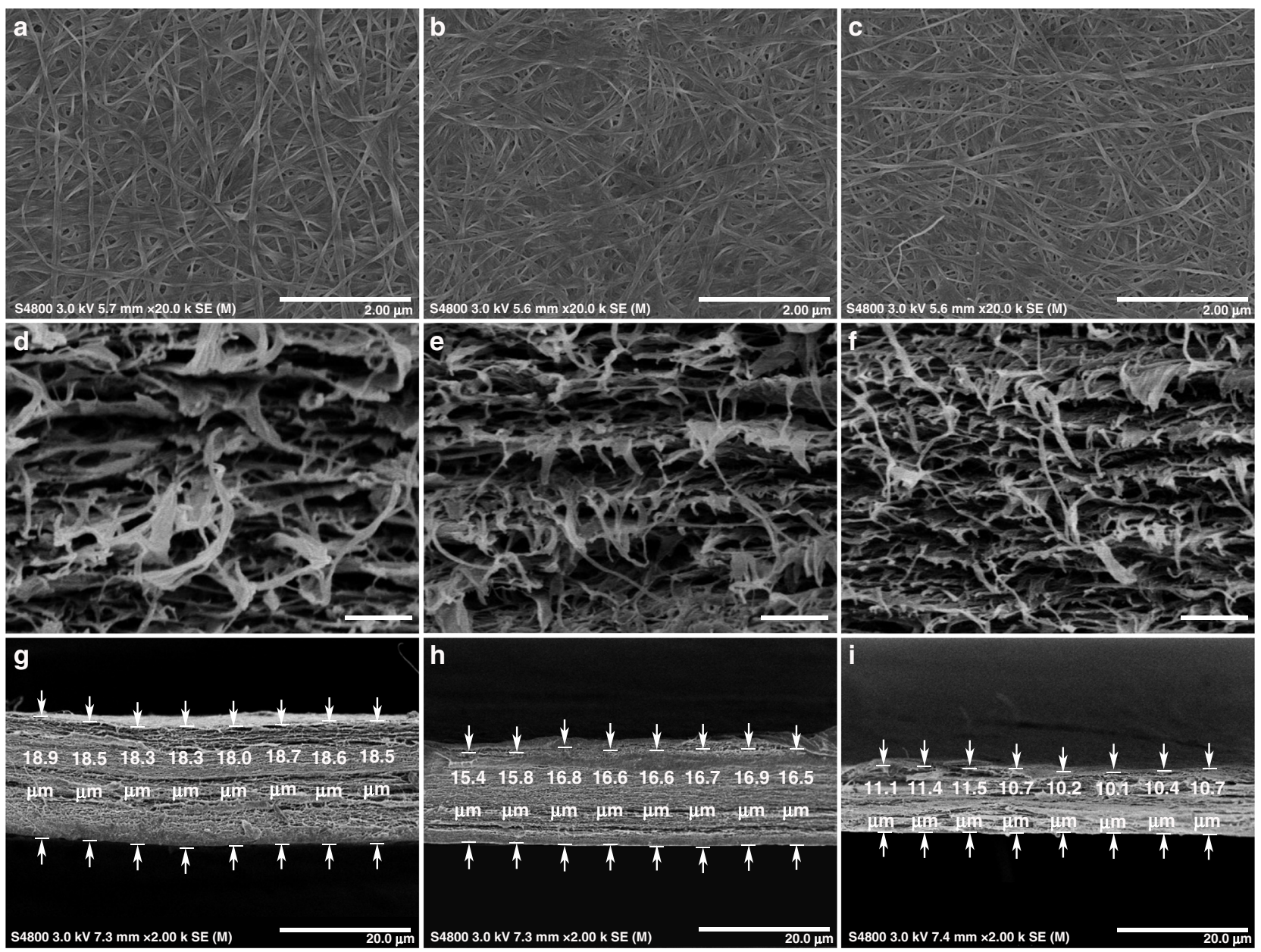

Fig. 5 Microstructure observation of $B C$ through SEM. a-c Top view of different $B C$ samples. The diameters of different $B C$ fibers showed no significant differences, whereas the pore sizes of $\mathbf{a}$ HC-6CF-BC and $\mathbf{b}$ LC-6CF-BC were slightly larger than that of $\mathbf{c} B C$. The scale bar corresponds to $2 \mu \mathrm{m}$. $\mathbf{d}-\mathbf{i}$ Crosssection of different BC samples. The gaps of $\mathbf{d}$ HC- 6 CF-BC were significantly larger than those of $\mathbf{e} \mathrm{LC}-6 \mathrm{CF}-\mathrm{BC}$ and $\mathbf{f} \mathrm{BC}$ samples. Moreover, $\mathbf{g} \mathrm{HC}-6 \mathrm{CF}-\mathrm{BC}$ was also thicker than $\mathbf{h}$ LC-6CF-BC and $\mathbf{i}$ BC samples. $\mathbf{d}-\mathbf{f}$, The scale bar corresponds to $400 \mathrm{~nm}$. $\mathbf{g}-\mathbf{i}$, The scale bar corresponds to $20 \mu \mathrm{m}$

microorganisms can metabolize and utilize the modified glucose (6CF-Glc) but also provided a method for the in situ synthesis of functional materials and possibly functional drugs in other microbial synthetic systems.

\section{Methods}

Strains and culture medium. K. sucrofermentans was used for BC fermentation. $\mathrm{H}-\mathrm{S}$ basic medium $\left(\mathrm{g} \mathrm{L}^{-1}\right)$ consisted of glucose 25 , yeast extract 5 , peptone 5 , citric acid monohydrate 1.2 and $\mathrm{Na}_{2} \mathrm{HPO}_{4}$ 2.7. The volume of all fermentation media was constant $(40 \mathrm{~mL})$, and they were sterilized at $115^{\circ} \mathrm{C}$ for $30 \mathrm{~min}$.

Synthesis of 6CF-GIc. The synthesis method was based on a previous study ${ }^{30}$, with slight modifications. Dried N,N-dimethylformamide (DMF, $3 \mathrm{~mL}$ ) was used as solvent for synthesis of the 6CF-Glc conjugate to dissolve $50 \mathrm{mg}$ of $6 \mathrm{CF}$ (AAT Bioquest, Inc., USA). Subsequently, $20 \mathrm{mg}$ of $\mathrm{N}, \mathrm{N}, \mathrm{N}^{\prime}, \mathrm{N}^{\prime}$-tetramethyl-O-(N-succinimidyl) uronium tetrafluoroborate (Macklin Biochemical Co., Ltd., Shanghai, China) and $100 \mu \mathrm{L}$ of N,N-diisopropylethylamine (DIPEA, Macklin Biochemical Co., Ltd., Shanghai, China) were added and incubated for $30 \mathrm{~min}$ at room temperature under inert conditions to activate 6CF. Dried DMF with DIPEA $(100 \mu \mathrm{L})$ was used to dissolve $25 \mathrm{mg}$ of $1,3,4,6$-tetra-O-acetyl-2-amino-2-deoxy- $\beta$-D-glucopyranose hydrochloride (Aladdin, Shanghai, China) as parallel-group. The 1,3,4,6-tetra-O-acetyl-2-amino-2-deoxy- $\beta$-D-glucopyranose solution was mixed with activated $6 \mathrm{CF}$ solution and incubated overnight at room temperature under inert and dark conditions. The product was an orange solid. Then, column chromatography (silica) and thin-layer chromatography (dichloromethane/MeOH 10:1) were applied to remove excess coupling reagents and elute the product, respectively. A solution of $\mathrm{NaOH}(3 \mathrm{~mL}, 0.01 \mathrm{M})$ was added to $15 \mathrm{mg}$ of the orange conjugate for deacetylation. The solution was left to react for $10 \mathrm{~min}$ at room temperature after ultrasonicated for $5 \mathrm{~s}$. Thin-layer chromatography (dichloromethane/MeOH 10:1) was used to monitor the extent of deacetylation. $\mathrm{HCl}(3 \mathrm{~mL}, 0.01 \mathrm{M})$ was applied to stop the reaction by neutralizing the mixture to $\mathrm{pH}$ 7.0. At last, the target product was obtained by vacuum drying and characterized by UV-Vis spectroscopy.

Microbial fermentation. K. sucrofermentans was inoculated in 7\% (v/v) H-S basic medium and cultured at $30^{\circ} \mathrm{C}$ for 5 days. The obtained $\mathrm{BC}$ samples were treated with sodium hydroxide solution $(2 \%, w / v)$ overnight at $60^{\circ} \mathrm{C}$, and then washed thoroughly with deionized water. All samples were dried at $60^{\circ} \mathrm{C}$ in a constant temperature drying oven.

The selectivity of $6 \mathrm{CF}$ on $\boldsymbol{K}$. sucrofermentans. $K$. sucrofermentans was cultured with $\mathrm{H}$-S basic medium supplemented with $6 \mathrm{CF}(50 \mathrm{mg})$ at $30^{\circ} \mathrm{C}$ for 5 days. Then, the obtained $\mathrm{BC}$ was washed thoroughly with deionized water.

Preparation of 6CF-BC. K. sucrofermentans was cultured with H-S basic medium supplemented with $6 \mathrm{CF}-\mathrm{Glc}\left(0.38 \mathrm{mg} \mathrm{mL}^{-1}\right.$ or $\left.0.95 \mathrm{mg} \mathrm{mL}^{-1}\right)$ at $30^{\circ} \mathrm{C}$ for 5 days. Then, $6 \mathrm{CF}-\mathrm{BC}$ was treated with sodium hydroxide solution $(2 \%, \mathrm{w} / \mathrm{v})$ overnight at $60^{\circ} \mathrm{C}$ and then washed with deionized water thoroughly until no fluorescence was detected in the residual water. All samples were dried at $60^{\circ} \mathrm{C}$ in a constant temperature drying oven.

Preparation of Ch-6CF/BC. The preparation method was based on a previous study ${ }^{29}$, with slight modifications. BC was produced by microbial fermentation under standard conditions mentioned above without 6CF-Glc. N,N-dimethylacetamide (DMF) was selected as the solvent for 6CF because of its poor solubility in water. $6 \mathrm{CF}(50 \mathrm{mg})$ was mixed with dried DMF $(20 \mathrm{~mL}), \mathrm{N}, \mathrm{N}, \mathrm{N}^{\prime}, \mathrm{N}^{\prime}$-tetramethyl -O(N-succinimidyl) uronium tetrafluoroborate $(20 \mathrm{mg})$ and DIPEA $(100 \mu \mathrm{L})$ and left 


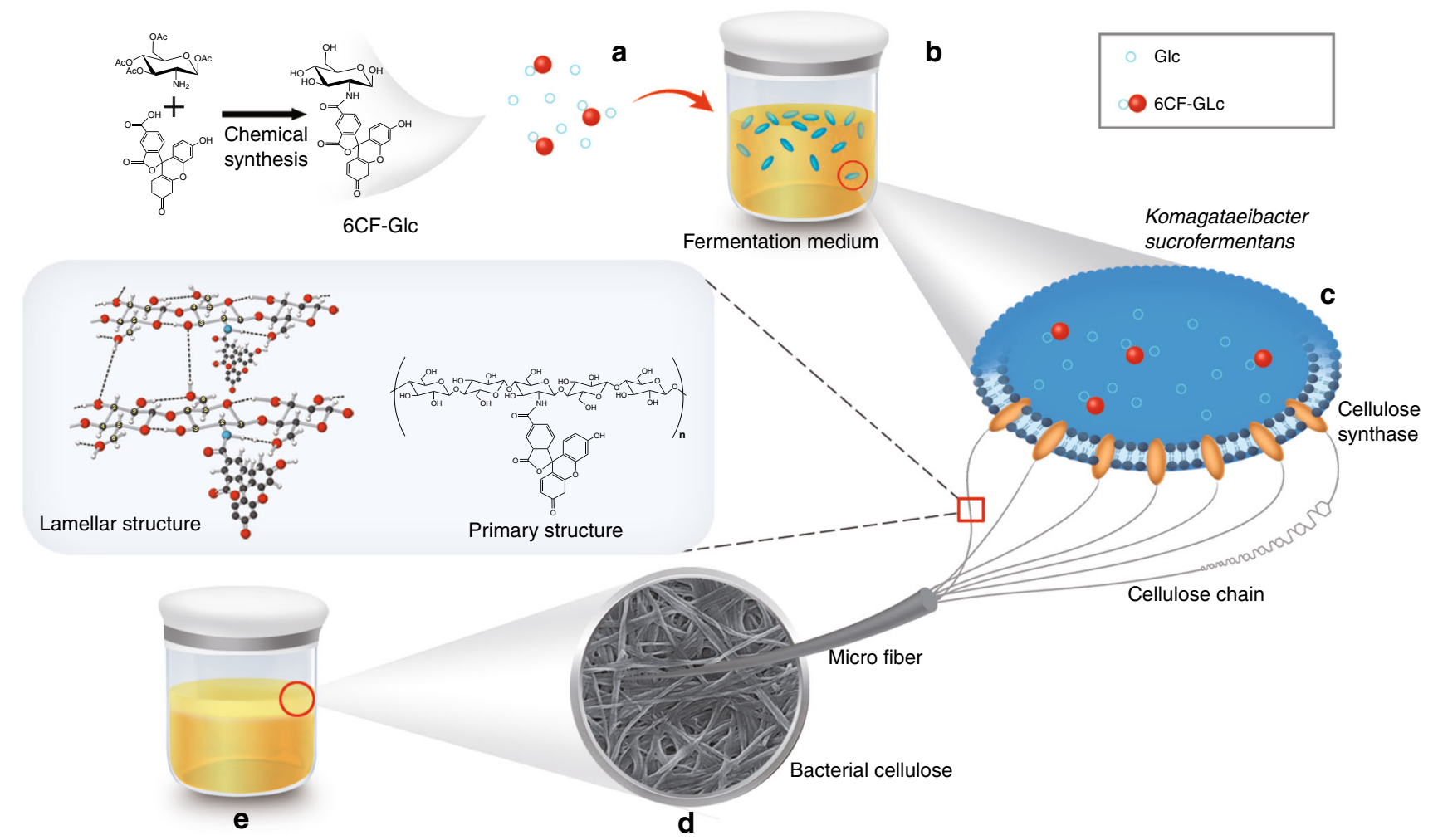

Fig. 6 Synthesis of 6CF-BC based on an in situ microbial fermentation method. Glucose (Glc) was modified with 6CF, and the 6CF-Glc was used as a carbon source for $\mathrm{K}$. sucrofermentans fermentation to obtain 6CF-BC through microbial metabolic pathways. a Glc and 6CF-Glc molecules. b Microorganism fermentation. c The synthesis of 6CF-BC fibers through K. sucrofermentans. d Microstructure of 6CF-BC. e The 6CF-BC pellicle obtained through microorganism fermentation

to activate for $30 \mathrm{~min}$ at room temperature under inert conditions. Then, neat BC was soaked in activated 6CF DMF solution for $30 \mathrm{~min}$ at room temperature under inert conditions and dried at $80^{\circ} \mathrm{C}$. Next, the chemically modified BC (Ch-6CF/BC) was washed with excess DMF and dried at room temperature.

Preparation of Im-6CF/BC. Neat BC was soaked in the H-S basic medium supplemented with $6 \mathrm{CF}-\mathrm{Glc}\left(0.95 \mathrm{mg} \mathrm{mL}^{-1}\right)$ at $30^{\circ} \mathrm{C}$ and allowed to physically adsorb for 5 days. Then, the obtained BC (Im-6CF/BC) was treated with sodium hydroxide solution $(2 \%, w / v)$ overnight at $60^{\circ} \mathrm{C}$ and washed with deionized water until no fluorescence was detected in the residual water. All samples were dried at $60{ }^{\circ} \mathrm{C}$ in a constant temperature drying oven.

CLSM. CLSM (FluoView FV1000, Olympus, Japan) was used for observing different BC fibers. Images were recorded at $488 \mathrm{~nm}$ (excitation) and $515 \mathrm{~nm}$ (emission).

FT-IR ATR. FT-IR spectroscopic measurements of glucose, 6CF-Glc, BC, Im-6CF/ $\mathrm{BC}$, and $6 \mathrm{CF}-\mathrm{BC}$ were recorded on a Nicolet 6700 FTIR Spectrometer (Thermo Fisher, USA) at $25^{\circ} \mathrm{C}$ and $30 \%$ relative humidity. All spectra were recorded in the spectral range between 700 and $4000 \mathrm{~cm}^{-1}$ with a resolution of $4 \mathrm{~cm}^{-1}$, averaging 32 scans. The experiment was repeated at least three times.

${ }^{1}$ H NMR. ${ }^{1} \mathrm{H}$ NMR spectra were recorded using the AVANCE-III 600 NMR spectrometer (600 MHz, Bruker BioSpin, Switzerland). 6CF-Glc was dissolved in $\mathrm{CD}_{3} \mathrm{OD}$. Data were processed using MestRENova (v.12.0.2, MestRElab Research, Spain).

Liquid chromatography-mass spectrometry (LC-MS). UHPLC analysis was carried out using an Ultimate 3000 UHPLC (Thermo, USA) with a Thermo Acclaim RSLC C18 column $(2.1 \mathrm{~mm} \times 100 \mathrm{~mm}, 2.2 \mu \mathrm{m})$, and Thermo online UHPLC filter $(2.1 \mathrm{~mm}, 0.2 \mu \mathrm{m})$ was used for the chromatographic separation. The mobile phase A consisted of $0.1 \%$ of formic acid in water, and the mobile phase B was composed of $\mathrm{CH}_{3} \mathrm{CN}$ with $0.1 \%$ formic acid. The elution gradient was started at $2 \% \mathrm{~B}$ for the first $2 \mathrm{~min}$ with flow rate of $0.2 \mathrm{~mL} \mathrm{~min}^{-1}$, stepping to $40 \% \mathrm{~B}$ at $6 \mathrm{~min}$, holding at $40 \% \mathrm{~B}$ until $10 \mathrm{~min}$, and returning to $2 \% \mathrm{~B}$ at $10.1 \mathrm{~min}$, holding these conditions at $15 \mathrm{~min}$ and stopping the controller. The injection volume was $5 \mu \mathrm{L}$ and the temperature of column was $30^{\circ} \mathrm{C}$

A Compact Q-TOF mass spectrometry (Bruker Daltonics, Billerica, USA) with an ESI source in negative ion mode with HyStar 3.2 software (Bruker Daltonics, Billerica, USA) was used to link the LC and the MS, using the following operation parameters: capillary voltage $3800 \mathrm{~V}$, dry temperature $200^{\circ} \mathrm{C}$, nebulizing gas of 1.5 bar, drying gas $\left(\mathrm{N}_{2}\right.$, purity $\left.99.99 \%\right)$ flowing of $4.5 \mathrm{~L} \mathrm{~min}^{-1}$. High-resolution MS and MS/MS spectra were acquired in the range $50-1300 \mathrm{~m} / \mathrm{z}$. The collision gas was high purity nitrogen $(99.99 \%)$. The data were collected by auto MS/MS acquisition with a MS scan rate of 1 spectra $\mathrm{s}^{-1}$ and MS/MS scan rate of three precursor was acquired per cycle, active exclusion after 3 spectra and $1.0 \mathrm{~min}$. Otof Control software (Bruker Daltonics, Billerica, USA) was used to carry out mass spectrometer control and data acquisition, and Compass Data Analysis software (Bruker Daltonics, Billerica, USA) was applied for data analysis.

XRD. The processed dried sample was analyzed on a D8 Advance diffractometer (Bruker, Germany) using $\mathrm{Cu} K a$ radiation $(\lambda=0.154 \mathrm{~nm})$ at $40 \mathrm{kV}$ and $40 \mathrm{~mA}$ with a scanning range of $5-50^{\circ}$. The experiment was repeated at least three times.

TG analysis. The measurement was performed on a thermogravimetry analyzer (STA449F5 Jupiter, Netzsch, Germany). The samples (2-3 mg) were weighed accurately into aluminum pans and sealed. The aluminum pans were scanned from $25^{\circ} \mathrm{C}$ to $600^{\circ} \mathrm{C}$ with heating rates of $10^{\circ} \mathrm{C} \mathrm{min}^{-1}$ under a $\mathrm{N}_{2}$ atmosphere $\left(50 \mathrm{~mL} \mathrm{~min}{ }^{-1}\right)$. For the buoyancy correction, a baseline was recorded using approximately $40 \mathrm{mg}$ of dry $\mathrm{Al}_{2} \mathrm{O}_{3}$ and subtracted from the sample measurements. The data were analyzed using Origin Lab 8 (Origin Lab Corporation, USA). The experiment was repeated at least three times.

SEM observation. The morphologies of $\mathrm{BC}, 6 \mathrm{CF}-\mathrm{BC}$ and $\mathrm{Im}-6 \mathrm{CF} / \mathrm{BC}$ samples were observed using an S-4800 SEM (Hitachi, Japan). The samples were mounted onto an aluminum stub, coated by platinum sputter and analyzed using SEM with a $3.0 \mathrm{kV}$ acceleration voltage.

Tensile testing. The dried $\mathrm{BC}$ and $6 \mathrm{CF}-\mathrm{BC}$ samples were cut into ribbons $(1 \mathrm{~cm} \times$ $10 \mathrm{~cm}$ ). Each sample was fixed at both ends of the machine grips. An Instron universal tester 5966 (Instron, Norwood, MA, USA) was used to apply 
unidirectional tension while recording the force and extension. The loading rate was maintained at $10 \mathrm{~mm} \mathrm{~min}^{-1}$. The tensile fracture stress was determined as the values obtained from the initial linear section of the stress-strain curves. The experiment was repeated at least six times.

Statistics. Dots in figures report values obtained from independent replicates unless otherwise noted. Data in tables are expressed as means \pm s.d. Statistical analyses were performed using SPSS Statistics 21.0 (IBM, New York, USA). Two-tailed Student $t$-tests were used in all comparisons. $P<0.05$ was considered statistically significant throughout the study.

\section{Data availability}

The data that support the findings of this study are available from the corresponding author upon reasonable request.

Received: 11 August 2018 Accepted: 19 November 2018

Published online: 25 January 2019

\section{References}

1. Abbasi-Moayed, S., Golmohammadi, H. \& Hormozi-Nezhad, M. R. A nanopaper-based artificial tongue: a ratiometric fluorescent sensor array on bacterial nanocellulose for chemical discrimination applications. Nanoscale 10, 2492-2502 (2018).

2. Yao, J., Ji, P., Wang, B., Wang, H. \& Chen, S. Color-tunable luminescent macrofibers based on CdTe QDs-loaded bacterial cellulose nanofibers for $\mathrm{pH}$ and glucose sensing. Sens. Actuat. B-Chem. 254, 110-119 (2018).

3. Wang, B. et al. Embedded binary functional materials/cellulose-based paper as freestanding anode for lithium ion batteries. Electrochim. Acta 260, 1-10 (2018).

4. Phiri, J., Johansson, L.-S., Gane, P. \& Maloney, T. C. Co-exfoliation and fabrication of graphene based microfibrillated cellulose composites-mechanical and thermal stability and functional conductive properties. Nanoscale 10, 9569-9582 (2018).

5. Li, Y. et al. Composites of bacterial cellulose and small molecule-decorated gold nanoparticles for treating gram-negative bacteria-infected wounds. Small 13, 1700130 (2017).

6. Ye, S. et al. Flexible amoxicillin-grafted bacterial cellulose sponges for wound dressing: in vitro and in vivo evaluation. ACS Appl. Mater. Inter. 10, 5862-5870 (2018).

7. Bäckdahl, H. et al. Mechanical properties of bacterial cellulose and interactions with smooth muscle cells. Biomaterials 27, 2141-2149 (2006).

8. Campano, C. et al. Enhancement of the fermentation process and properties of bacterial cellulose: a review. Cellulose 23, 57-91 (2016).

9. Hirai, A., Tsuji, M. \& Horii, F. TEM study of band-like cellulose assemblies produced by Acetobacter xylinum at $4{ }^{\circ} \mathrm{C}$. Cellulose 9, 105-113 (2002).

10. Ross, P., Mayer, R. \& Benziman, M. Cellulose biosynthesis and function in bacteria. Microbiol. Rev. 55, 35-58 (1991).

11. Czaja, W. K., Young, D. J., Kawecki, M. \& Brown, R. M. The future prospects of microbial cellulose in biomedical applications. Biomacromolecules 8, 1-12 (2007).

12. He, J. et al. Controlled incorporation of deuterium into bacterial cellulose. Cellulose 21, 927-936 (2014).

13. Reiniati, I., Hrymak, A. N. \& Margaritis, A. Recent developments in the production and applications of bacterial cellulose fibers and nanocrystals. Crit. Rev. Biotechnol. 37, 510-524 (2017).

14. Klemm, D., Heublein, B., Fink, H. P. \& Bohn, A. Cellulose: fascinating biopolymer and sustainable raw material. Angew. Chem. Int. Ed. 44, 3358-3393 (2005).

15. Yamanaka, S. et al. The structure and mechanical properties of sheets prepared from bacterial cellulose. J. Mater. Sci. 24, 3141-3145 (1989).

16. Miyamoto, T., Takahashi, Si, Ito, H., Inagaki, H. \& Noishiki, Y. Tissue biocompatibility of cellulose and its derivatives. J. Biomed. Mater. Res. 23, 125-133 (1989).

17. Klemm, D., Schumann, D., Udhardt, U. \& Marsch, S. Bacterial synthesized cellulose - artificial blood vessels for microsurgery. Prog. Polym. Sci. 26, 1561-1603 (2001).

18. Lee, K.-Y. et al. Surface only modification of bacterial cellulose nanofibres with organic acids. Cellulose 18, 595-605 (2011).

19. He, J. et al. Superelastic and superhydrophobic bacterial cellulose/silica aerogels with hierarchical cellular structure for oil absorption and recovery. $J$. Hazard. Mater. 346, 199-207 (2018)

20. Hou, Y. et al. Development and biocompatibility evaluation of biodegradable bacterial cellulose as a novel peripheral nerve scaffold. J. Biomed. Mater. Res. A. 106, 1288-1298 (2018).
21. Lv, P. et al. Self-assembly of nitrogen-doped carbon dots anchored on bacterial cellulose and their application in iron ion detection. Carbohydr. Polym. 172, 93-101 (2017).

22. Zhang, $\mathrm{X}$. et al. Ultrafine nano-network structured bacterial cellulose as reductant and bridging ligands to fabricate ultrathin K-birnessite type $\mathrm{MnO}_{2}$ nanosheets for supercapacitors. Appl. Surf. Sci. 433, 419-427 (2018).

23. Smith, C. J. et al. Bacterial cellulose ionogels as chemosensory supports. ACS Appl. Mater. Inter. 9, 38042-38051 (2017).

24. Lv, P. et al. Copper nanoparticles-sputtered bacterial cellulose nanocomposites displaying enhanced electromagnetic shielding, thermal, conduction, and mechanical properties. Cellulose 23, 3117-3127 (2016).

25. $\mathrm{Li}, \mathrm{S}$. et al. Freestanding bacterial cellulose-polypyrrole nanofibres paper electrodes for advanced energy storage devices. Nano Energy 9, 309-317 (2014).

26. Medronho, B., Romano, A., Miguel, M. G., Stigsson, L. \& Lindman, B. Rationalizing cellulose (in) solubility: reviewing basic physicochemical aspects and role of hydrophobic interactions. Cellulose 19, 581-587 (2012).

27. Sen, S., Martin, J. D. \& Argyropoulos, D. S. Review of cellulose nonderivatizing solvent interactions with emphasis on activity in inorganic molten salt hydrates. ACS Sustain. Chem. Eng. 1, 858-870 (2013).

28. Wang, S., Lu, A. \& Zhang, L. Recent advances in regenerated cellulose materials. Prog. Polym. Sci. 53, 169-206 (2016).

29. Chen, H. et al. Citric acid/cysteine-modified cellulose-based materials: Green preparation and their applications in anticounterfeiting, chemical sensing, and UV shielding. ACS Sustain. Chem. Eng. 5, 11387-11394 (2017).

30. Natalio, F. et al. Biological fabrication of cellulose fibers with tailored properties. Science 357, 1118-1122 (2017).

31. Becker, J. \& Wittmann, C. Advanced biotechnology: metabolically engineered cells for the bio-based production of chemicals and fuels, materials, and health-care products. Angew. Chem. Int. Ed. 54, 3328-3350 (2015).

32. Natalio, F. et al. Structural analysis of Gossypium hirsutum fibers grown under greenhouse and hydroponic conditions. J. Struct. Biol. 194, 292-302 (2016).

33. Abidi, N. et al. Evaluating cell wall structure and composition of developing cotton fibers using Fourier transform infrared spectroscopy and thermogravimetric analysis. J. Appl. Polym. Sci. 107, 476-486 (2008).

34. Yilgör, E., Burgaz, E., Yurtsever, E. \& Yilgör, İ. Comparison of hydrogen bonding in polydimethylsiloxane and polyether based urethane and urea copolymers. Polym. (Guildf.). 41, 849-857 (2000).

35. Araújo, I. M. et al. Hydrothermal synthesis of bacterial cellulose-copper oxide nanocomposites and evaluation of their antimicrobial activity. Carbohydr. Polym. 179, 341-349 (2018).

36. de Lima Fontes, M. et al. Effect of in situ modification of bacterial cellulose with carboxymethylcellulose on its nano/microstructure and methotrexate release properties. Carbohydr. Polym. 179, 126-134 (2018).

37. Mwaikambo, L. Y. \& Ansell, M. P. Chemical modification of hemp, sisal, jute, and kapok fibers by alkalization. J. Appl. Polym. Sci. 84, 2222-2234 (2002).

38. Lizundia, E., Maceiras, A., Vilas, J., Martins, P. \& Lanceros-Mendez, S. Magnetic cellulose nanocrystal nanocomposites for the development of green functional materials. Carbohydr. Polym. 175, 425-432 (2017).

39. Gescher, A. Metabolism of N, N-dimethylformamide: key to the understanding of its toxicity. Chem. Res. Toxicol. 6, 245-251 (1993).

40. Lee, K. Y., Buldum, G., Mantalaris, A. \& Bismarck, A. More than meets the eye in bacterial cellulose: biosynthesis, bioprocessing, and applications in advanced fiber composites. Macromol. Biosci. 14, 10-32 (2014).

41. Thongsomboon, W. et al. Phosphoethanolamine cellulose: A naturally produced chemically modified cellulose. Science 359, 334-338 (2018).

42. Achmon, Y., Zelas, Z. B.-B. \& Fishman, A. Cloning Rosa hybrid phenylacetaldehyde synthase for the production of 2-phenylethanol in a whole cell Escherichia coli system. Appl. Microbiol. Biotechnol. 98, 3603-3611 (2014).

43. Jenck, J. F., Agterberg, F. \& Droescher, M. J. Products and processes for a sustainable chemical industry: a review of achievements and prospects. Green. Chem. 6, 544-556 (2004).

\section{Acknowledgements}

This work is supported by National Natural Science Foundation of China (NSF No. 31400084), Youth Innovation Promotion Association CAS (No. 2017252), Hainan's Key Project of Research and Development Plan (NO. ZDYF2017155), and Taishan Scholars Climbing Program of Shandong (No. TSPD20150210). We thank Dr. Xinglin Jiang of Technical University of Denmark for reading the manuscript and providing many valuable comments. We thank Dr. Wenjie Gao and Dr. Zongjiang Yu of Qingdao Institute of Bioenergy and Bioprocess Technology, Chinese Academy of Sciences for their contribution in data analysis.

\section{Author contributions}

H.B.Z., M.X., and M.H.G. designed the project. M.H.G. and Z.X.B. wrote the first version of manuscript and designed all the figures. All the authors contributed to the final 
version. J.L. and Z.X.B. synthesized and characterized the 6CF-BC. J.L., Y.D.H., and D.A. synthesized and characterized the 6CF-Glc. J.L., R.N., and D.X.F. performed the tensile assays. Xing Li performed the microbial species identification.

\section{Additional information}

Supplementary Information accompanies this paper at https://doi.org/10.1038/s41467018-07879-3.

Competing interests: The authors declare no competing interests.

Reprints and permission information is available online at http://npg.nature.com/ reprintsandpermissions/

Publisher's note: Springer Nature remains neutral with regard to jurisdictional claims in published maps and institutional affiliations. (c) (i) Open Access This article is licensed under a Creative Commons Attribution 4.0 International License, which permits use, sharing, adaptation, distribution and reproduction in any medium or format, as long as you give appropriate credit to the original author(s) and the source, provide a link to the Creative Commons license, and indicate if changes were made. The images or other third party material in this article are included in the article's Creative Commons license, unless indicated otherwise in a credit line to the material. If material is not included in the article's Creative Commons license and your intended use is not permitted by statutory regulation or exceeds the permitted use, you will need to obtain permission directly from the copyright holder. To view a copy of this license, visit http://creativecommons.org/ licenses/by/4.0/.

(C) The Author(s) 2019 